\title{
EVOLUTION OF CRITICALLY ILL GERIATRIC PATIENTS WITH HYPERTENSIVE EMERGENCIES IN THE YAOUNDE EMERGENCY CENTER
}

\author{
Nga Nomo $S^{1}$, Kuitchet $A^{3}$, Iroume $C^{4}$, Chewa ${ }^{1}$, Zoumenou E4, Chobli $M^{4}$ \\ 1. Service d'Anesthésie-Réanimation, Centre Hospitalier d'Essos/Yaoundé \\ 2. Service d'Anesthésie-Réanimation, Hôpital régional de Maroua \\ 3. Service d'Anesthésie-Réanimation, Centre Hospitalier Universitaire de Yaoundé \\ 4. Département d'anesthésie-Réanimation, Faculté des sciences de la santé de Cotonou/Bénin
}

\section{Email : sergevivier@yahoo.fr}

\begin{abstract}
Introduction: Aging is an inevitable part of life, and it brings along two inconvenient events: physiologic decline and disease state [1]. Over 1 billion people worldwide have hypertension, $40 \%$ of whom are adults older than $25 y e a r s$, which implies its prevalence increases further with age [6]. Our study focused on (hypertensive emergencies) HTN-E only, with general objective to describe how health factors influence the evolution of geriatric hypertensive emergencies in the intensive care unit (ICU) of the Yaounde emergency center. Materials and methods: This was a quantitative, descriptive, prospective, and cross-sectional study. The study lasted for 06 months, from January to June 2018. Data collection spanned from April to May 2018. The inclusion criteria were: the patients aged 60 years and above, admitted in the ICU for hypertensive emergencies within April to May 2018, the patients who consented to the study, the elderly patients with complete medical records. Results: Our sample was dominated by young-old patients, with a percentage of $73.3 \%(n=22)$. We find a predominance of the female sex $(53,3 \%)$ over the male sex $(46,7 \%)$. Majority of patients had been hypertensive for $1-5 y e a r s$ $46.7 \%(n=14)$, followed by $20 \%(n=6)$ patients who were not aware of their hypertensive status until they had a HTN-E. There was an equal percentage $16.7 \%(n=5)$ of patients who had been hypertensive for 6-10years and for 11-15years. The duration of hypertension was similar to the duration of antihypertensive treatment. A proportion of $60 \%(n=18)$ patients consumed 1 to 2 antihypertensives daily, while $26,7 \%(n=8)$ patients were not on any antihypertensives. The smallest proportion of patients $13,3 \%(n=4)$ was placed on 3-4 antihypertensives daily. Out of the 30 patients, majority of them $43.3 \%(n=13)$ hardly consumed their antihypertensive medications; $26.7 \% \quad(n=8)$ never consumed antihypertensives, $16.7 \%(n=5)$ consumed their medications sometimes, and the least proportion was $13.3 \%(n=4)$ of patients who always consumed their antihypertensive medication. Among the 30 patients with hypertensive emergencies, $30 \%$ ( $n=9$ ) had no comorbidities, while $70 \%(n=21)$ had at least, one comorbidity. Diabetes was the most prominent comorbidity with a prevalence of $23.3 \%(n=7)$, and then $16.7 \%(n=5)$ had respiratory diseases. More than half, $56.7 \%(n=17)$ of the patients had stroke, followed by $23 \%$ ( $n=7$ ) with acute pulmonary oedema, then equal percentages $10 \%$ ( $n=3$ each) for patients with coronary ischaemia and myocardial infarction. Conclusion: The hypertensive emergency syndromes encountered were neurologic deficit (stroke $56.7 \%$ )
\end{abstract}


and cardiovascular deficits (43.3\%). Our results revealed higher chances of death with higher APACHE II scores and more chances of discharge in case of low APACHE II score.

Keys words : critically ill, Geriatric patients, hypertensive emergencies. 


\section{Introduction}

Aging is an inevitable part of life, and it brings along two inconvenient events: physiologic decline and disease state [1]. Recently, there has been a sharp increase in the number of older persons worldwide, with an increasing average age and life expectancy of the population. As a result, a growing number of older persons are being admitted in the intensive care unit (ICU) [1]. They also have an increased risk of chronic diseases like hypertension. An elderly person can be defined as one who is aged 60 years or more. According to the World Health Organisation (WHO), elderly persons can be grouped into five categories as follows : the young-old (60-74years); the middleaged old (75-80years); the aged (8189years); the chronically-old (90years) and the oldest-old (above 90years) [4]. Intensive or critical care medicine is a medical specialty which focuses on the management of critically ill patients. A critically ill patient is one who has a medical pathology (such as stroke, trauma, infection, acute coronary syndrome among others) which causes the impairment of vital functions (consciousness, circulation, and respiration) or single organ functions (such as kidney and liver functions) [5]. Hypertension is a chronic medical condition, defined as a blood pressure (BP) reading greater than or equal to $130 / 80 \mathrm{mmHg}$, as opposed to greater than or equal to $140 / 90 \mathrm{mmHg}$ used in the past and still used in our setting (The American Heart Association, AHA 2017). Over 1 billion people worldwide have hypertension, $40 \%$ of whom are adults older than 25years, which implies its prevalence increases further with age [6]. One of the biggest health concerns in
Africa at large and Cameroon in particular is hypertension, with an increasing prevalence of $46 \%$ and $31 \%$ respectively $[\mathbf{7 , 8 ]}$. It is a major risk factor for cardiovascular morbidity and mortality particularly in older persons [9]. This therefore explains why despite recent improvements in control rates in many developed countries, hypertension remains a major public health challenge [10]. Hypertension is often asymptomatic, thus its early detection and treatment can minimise the complications that arise from its poor control. It therefore requires optimal control and persistent adherence to prescribed medication to reduce the risk of complications [1]. Hypertension, if uncontrolled can lead to hypertensive crises. Hypertensive crises are clinical syndromes characterised by acute elevations in BP that results as complications of untreated or inadequately treated hypertension [8]. It is commonly encountered in the emergency department, operating room, postanaesthesia care unit and intensive care units. The most important factor that limits morbidity and mortality due to hypertensive crises is prompt and carefully considered therapy [8]. Hypertensive crises encompass two categories [12] : hypertensive urgency (HTN-U) and hypertensive emergency (HTN-E). HTN - E is diagnosed as a systolic BP greater than or equal to $180 \mathrm{mmHg}$ and/or a diastolic BP greater than or equal to $110 \mathrm{mmHg}$ with clinical or laboratory evidence of acute organ damage [10]. Whereas HTN-U can be diagnosed with same BP levels as HTN-E, but in the absence of target organ damage. Many previous studies have shown that hypertensive emergencies are associated with substantial morbidity 
and mortality compared to HTN-U. Our study focused on HTN-E only, with general objective to describe how health factors influence the evolution of geriatric hypertensive emergencies in the ICU of the Yaoundé Emergency Center.

\section{Material and methods}

This was a quantitative, descriptive, prospective, and cross-sectional study. The study lasted for 06 months, from January to June 2018. Data collection spanned from April to May 2018. The clinical research method was used in this study. The target population was all geriatric patients presenting with hypertensive emergencies during the stipulated period for data collection. The inclusion criteria were : the patients aged 60 years and above, admitted in the ICU for hypertensive emergencies within April to May 2018, the patients who consented to the study, the elderly patients with complete medical records. A wellstructured questionnaire was the data collection tool used for this study. It was interviewer-guided, as there were many parameters, which may not be well understood by the patients, or their carers (for unconscious patients). The questionnaire was filled-in using both information from the participants themselves, and from their medical records, particularly laboratory test results. It was divided into three sections as follows:

Socio-demographic data: age, sex, marital status, date of admission, profession, and carer.

Health Data: duration of illness, duration of treatment, number of antihypertensive medications, how often the drugs are taken, means of transportation to the hospital, BP level on admission, comorbidities, presenting signs and symptoms, type of hypertensive emergency, APACHE II score, promptness of treatment, and additional complications.

Evolution: discharge or death.

Hypertensive emergency was the only dependant variable in our study. Geriatric patients were diagnosed with HTN-E if they presented with a BP reading $\geq 180 / 110 \mathrm{mmHg}$ taken on both arms, associated with either clinical or laboratory evidence of neurologic, cardiovascular or renal target organ damage. The outcome of the dependant variable was influenced by the independent variables. The factors that influenced the evolution of HTN-E were the independent variables. It was responsible for the alteration of the dependent variable (hypertensive emergency). The four independent variables that were studied included: specific HTN-E syndromes, APACHE ॥ score, risk factors for hypertensive emergency, and the outcome (recovery or mortality) of HTN-E.

Specific hypertensive syndromes presented either as neurologic, cardiovascular, or renal deficits. Neurologic deficits were characterised by the following clinical signs and symptoms: an alteration in the level of consciousness, partial body weakness or numbness, blurred vision, ataxia, aphasia, migraine, asthenia, and convulsions among others. Cardiovascular deficits were predominantly characterised by: dyspnoea, severe chest pain, tachycardia and pedal oedema. No renal deficits were represented in our study, though they may include: oliguria, haematuria, and acute renal insufficiency among others. Further laboratory tests were done to confirm or laboratory tests were done 
infirm the specific HTN-E syndrome implicated.

The APACHE II score was measured within 24hours of hospital admission for all our participants. It comprises three components: the acute physiology score, age adjustment and the chronic health evaluation components. The acute physiology score was measured clinically using vital signs and laboratory test results. The blood gas analysis was not done in our setting, so it was assigned 0 to all patients, while the others were calculated for each patient. The age adjustment component required us to request the patient's age, and fit them into the age ranges given on the score. The chronic health evaluation component was obtained following the patient's medical history. The score was then calculated for each patient, and interpreted as recommended. The risk factors studied were: sex, noncompliance to antihypertensive medications, comorbidities, number of antihypertensive medications consumed, and unknown hypertensive status. Though age is normally a risk factor, it was not studied here because the study was a cross-sectional one involving only geriatric patients; other age groups were not studied as would be the case in a cohort study. Sex was noted on patient admission. Compliance to antihypertensives was detected by requesting the frequency of its consumption, and the number of antihypertensives consumed. Comorbidities were requested and also diagnosed upon patient's admission and during their hospital stay. Unknown hypertensive status was recorded for patients who only discovered their hypertensive status in the hospital following their HTN-E. The evolution of HTN-E was studied in terms of patient outcome: recovery or mortality. Recovery was recorded for patients who were either discharged from the hospital, or were transferred to a normal ward for a continuity of treatment. While mortality was recorded for patients who died during their hospital stay. Complications and disabilities were equally studied and recorded alongside data collection. Participants were followed up until they left the hospital. The limitations of the study are the small sample size and the monocentric nature of the study.

\section{Results}

\section{Demographic Data:}

Our sample was dominated by young-old patients (60-74years), with a percentage of $73.3 \%$ ( $n=22$ ), followed by an equal representation of the middle-aged old (75-80years) and the aged (81-89years) patients, $13.3 \% \quad(\mathrm{n}=4)$ each. The chronically old (90years) and the oldest old (> 90years) patients were not represented (figure 1). 


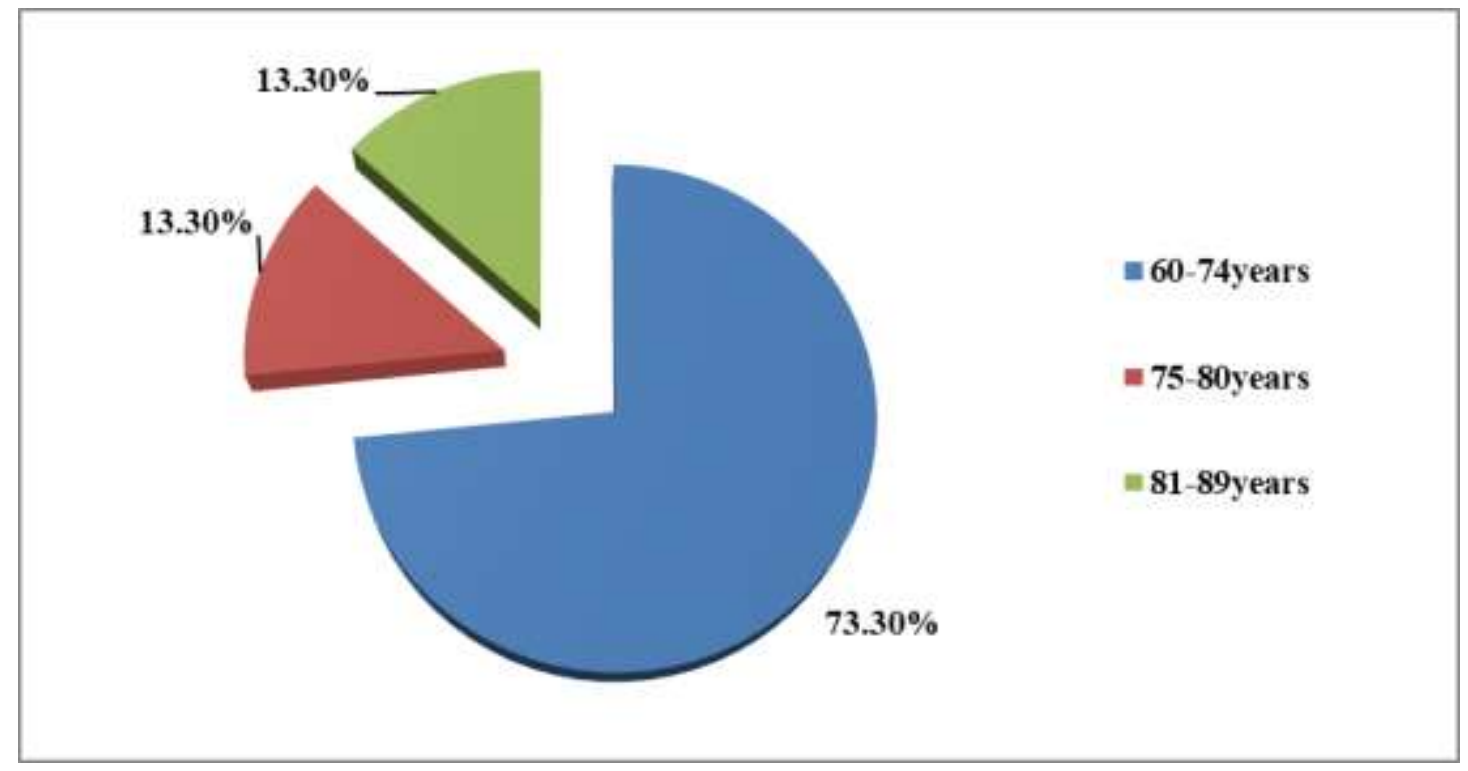

Figure 1 : Representation of Age Groups

In our series, we find a predominance of the female sex $(53,3 \%)$ over the male sex $(46,7 \%)$

Duration of Hypertension, Number of Antihypertensives Consumed and the Frequency of Consumption:

Majority of patients had been hypertensive for 1 -5years $46.7 \% \quad(n=14)$, followed by $20 \%(n=6)$ patients who were not aware of their hypertensive status until they had a HTN-E. There was an equal percentage $16.7 \%(n=5)$ of patients who had been hypertensive for 6-10years and for 11-15years (figure 2 )

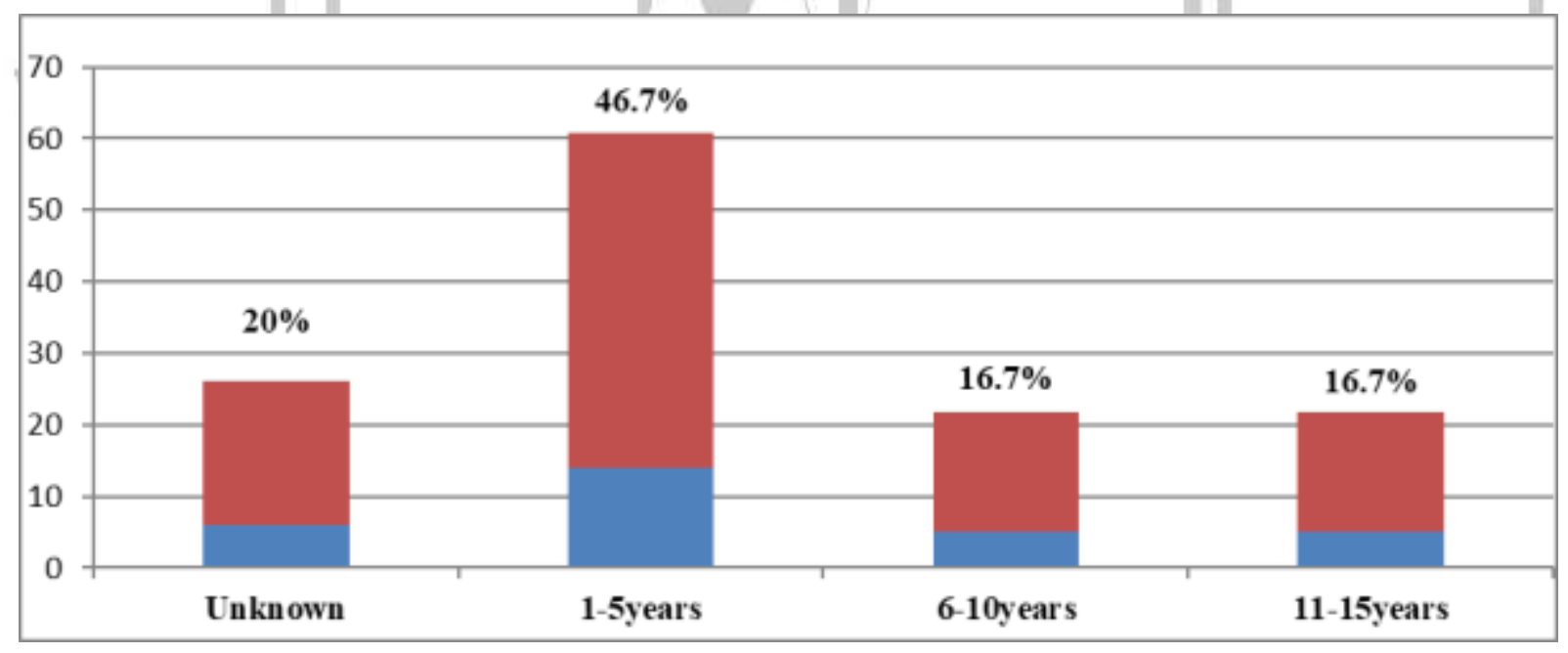

Figure 2: Duration of Hypertension

The duration of hypertension was similar to the duration of antihypertensive treatment. A proportion of $60 \% \quad(n=18)$ patients consumed 1 to 2 antihypertensives daily, while $26,7 \%(n=8)$ patients were not on any antihypertensives, despite the fact that 2 patients out of this number were aware of their hypertensive state. The smallest proportion of patients $13,3 \%(n=4)$ was placed on 3-4 antihypertensives daily. Out of the 30 patients, majority of them 
43.3\% ( $n=13)$ hardly consumed their antihypertensive medications; $26.7 \%(n=8)$ never consumed antihypertensives, $16.7 \%$ $(n=5)$ consumed their medications sometimes, and the least proportion was $13.3 \%(n=4)$ of patients who always consumed their antihypertensive medication (figure 3).

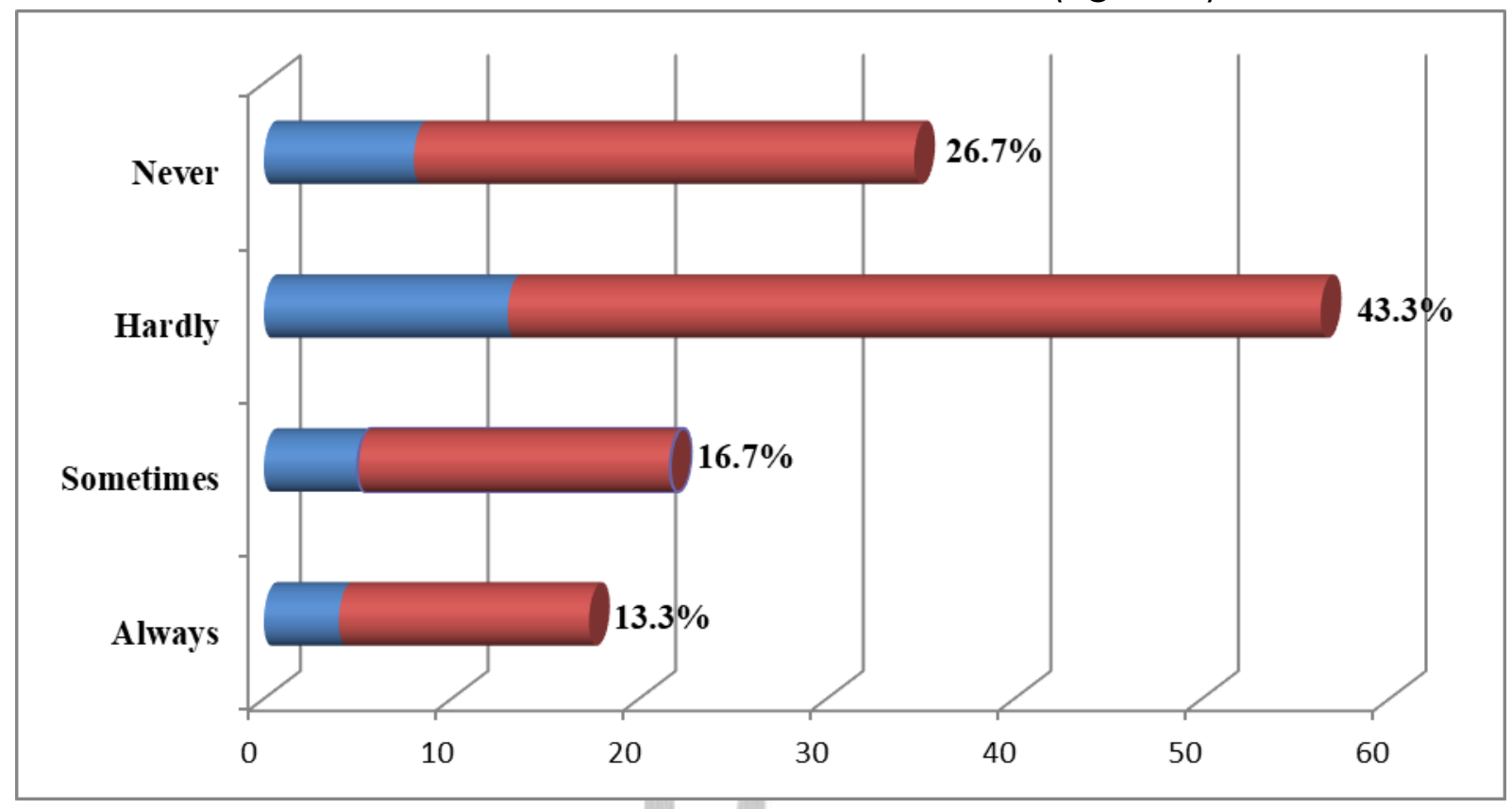

Figure 3: Frequency of Antihypertensive Medication Consumption

Among the 30 patients with hypertensive emergencies, $30 \%(n=9)$ had no comorbidities, while $70 \%(n=21)$ had at least, one comorbidity. The other comorbidities not mentioned on the chart (sepsis, ascites, obesity, HIV, alcoholism, and tobacco abuse) were predominant with a percentage of $30 \%(n=9)$. Diabetes was the most prominent comorbidity with a prevalence of $23.3 \%$ ( $n=7)$, and then $16.7 \%(n=5)$ had respiratory diseases (asthma, chronic bronchitis,

pneumonia, pneumopathy).

\section{Hypertensive Emergency Syndromes:}

More than half, $56.7 \%$ ( $n=17)$ of the 30 patients had stroke, followed by $23 \%$ ( $n=$ 7) with acute pulmonary oedema, then equal percentages $10 \%$ ( $n=3$ each) for patients with coronary ischaemia and myocardial infarction each. Therefore, only neurologic and cardiovascular HTN-E syndromes were represented. No renal HTN-E syndromes were represented (figure 4) 


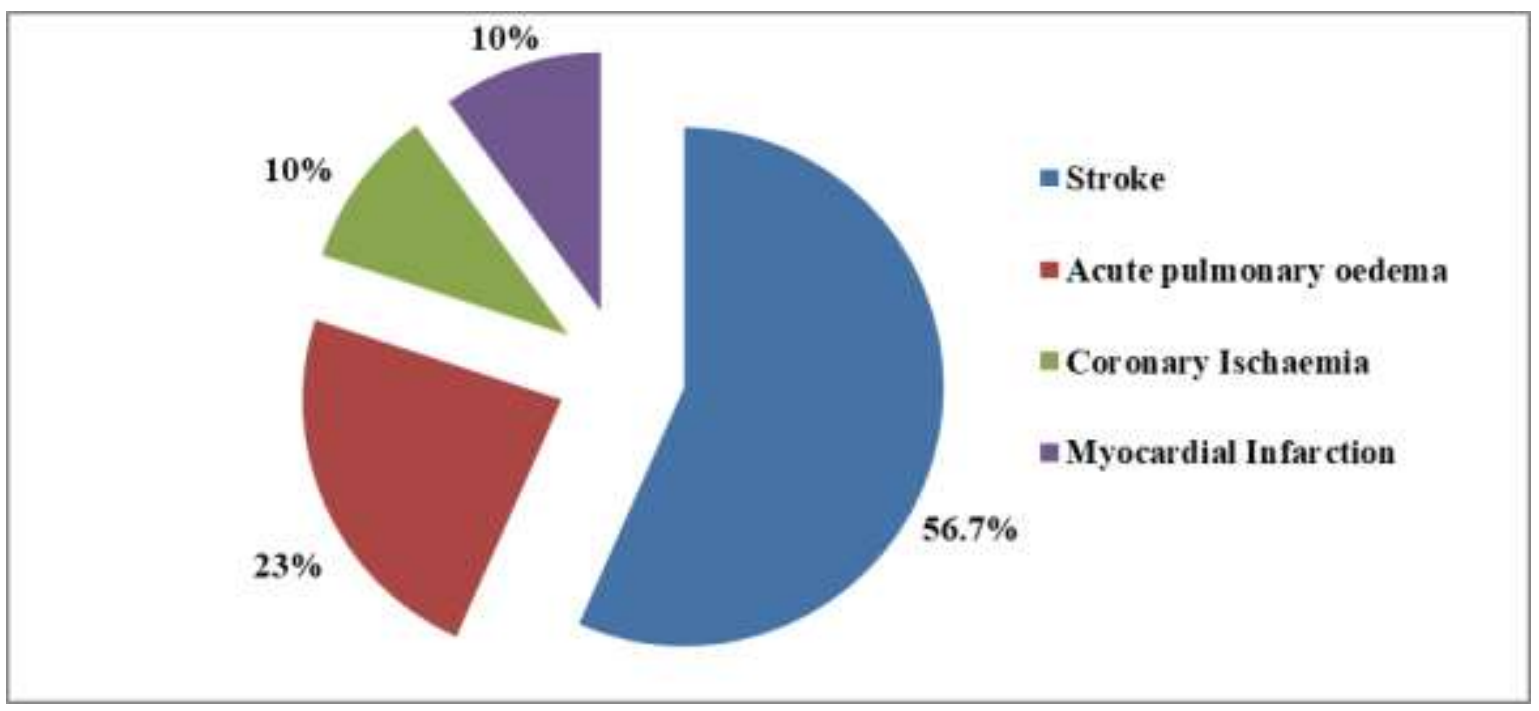

Figure 4: Specific Hypertensive Emergency Syndromes Encountered

\section{Prominent Signs and Symptoms Recorded:}

The most frequently encountered signs and symptoms were migraines and hemiparesis with $23.3 \%(n=7)$ each. These were followed by severe thoracic pain $16.7 \%(n=5)$, then dyspnoea and unconsciousness with $13.3 \%(n=4)$ each, and lastly $10 \%(\mathrm{n}=3$ ) for pedal (lower limbs) oedema. These symptoms were not exclusive, as we found more than one symptom in each patient. We presented just the most frequent signs and symptoms to enable an orderly presentation of our work. However, other associated symptoms included: difficult speech (aphasia), blurred vision, body weakness (asthenia), alteration in general state, convulsions, epistaxis and vomiting.

\section{The APACHE II Score:}

Based on the APACHE II score, the patients were divided into five groups: the first group of patients had APACHE II score of 3-10, the second group 11-20, third group 21-30, fourth group 31-40 and the fifth group had $>40$. The first group had the highest number of patients, 13 patients. Out of the 13 patients, 12 (92.3\%) were discharged and $01 \quad(7.7 \%)$ died. A proportion of 11 patients were in the second group, out of which $08(72.7 \%)$ survived and 03 (27.3\%) died. The third group comprised 06 patients, among which 01 (16.7\%) patient was discharged, and 05 (83.3\%) died (table 1)

\section{Table 1 : The APACHE II Score and Observed Mortality Rate}

\begin{tabular}{|l|l|l|l|l|l|}
\hline $\begin{array}{l}\text { APACHE II } \\
\text { Score }\end{array}$ & $\begin{array}{l}\text { Number of } \\
\text { Patients }\end{array}$ & $\begin{array}{l}\text { Patients } \\
\text { Discharged }\end{array}$ & Patients Died & $\begin{array}{l}\text { Observed } \\
\text { Mortality } \\
(\%)\end{array}$ & $\begin{array}{l}\text { Predicted } \\
\text { Mortality } \\
(\%)\end{array}$ \\
\hline $\mathbf{3 - 1 0}$ & 13 & 12 & 01 & 7.7 & 11 \\
\hline $\mathbf{1 1 - 2 0}$ & 11 & 08 & 03 & 27.3 & 35.5 \\
\hline $\mathbf{2 1 - 3 0}$ & 06 & 01 & 05 & 83.3 & 70.3 \\
\hline
\end{tabular}


Our results revealed higher chances of death with higher APACHE II scores and more chances of discharge in case of IOW APACHE II score. The mortality rate associated with hypertensive emergencies was $30 \%(n=9)$.

Complications (Morbidity) Resulting from Hypertensive Emergencies:

A half of the patient who were discharged had no significant disabilities. The most prominent disabilities were neurologic disabilities (gait disturbance, hemianopia, and seizures) with $23 \%$ ( $n=$ 7); followed by $16.7 \%(n=5)$ with cardiac disabilities (cardiac arrhythmia and cardiogenic shock, which is the major cause of death in patients hospitalized with MI). A proportion of $10 \%(n=3)$ had vascular disabilities (deep venous thrombosis, DVT and suspected pulmonary embolism, PE).

\section{Comparison between Health Outcome} and Hypertensive Emergency Syndromes: Though stroke was the predominant HTNE syndrome, it accounted for the least mortality rate of $22.2 \% \quad(n=2)$, and the highest recovery rate of $71.4 \%(n=15)$. On the other hand, the cardiovascular HTN-E syndromes $(n=13)$ had predominant mortality rates of $77.8 \%(n=7)$ and the least recovery rates of $28.6 \% \quad(n=6)$ despite their lower prevalence. This high mortality rate was principally due to APO, which was often associated with myocardial infarction and coronary ischaemia in two of our patients, both of whom died.

\section{Comparison between Health Outcome and Age Groups:}

The young-old patients (60-74years) were predominant (22), hence represented more than half of both the discharged patients $17(81 \%)$ and the patients that died 5 (55.6\%). An equal representation of recovery rate $2(9.5 \%)$ and mortality rate 2 (22.2\%) was recorded in both the middle-aged old patients (75-80years) and the aged patients (81-89years).

\section{Health Outcome with respect to Risk Factors:}

All our participants had at least one comorbidity. These comorbidities accounted for the highest mortality rate of $77.8 \%(n=7)$, followed by a nonadherence to antihypertensives $66.7 \%$ $(n=6)$, then the female sex $155.6 \% \quad(n=5)$ and lastly an unknown hypertensive status $33.3 \% \quad(n=3)$. The unknown hypertensive status was complimentary with a non-adherence to antihypertensives. They were not grouped together because it would be irrational to add those ignorant of their hypertensive status to those who are aware, but do not take their medications adequately.

\section{Discussion}

Prediction of the evolution of patients admitted in the ICU remains an area of great concern for health personnel as well as for patient's families [12]. The impact of this prediction weighs on different aspects of patient care like, the choice of medical therapy, the onset of medical treatment, risk factors, complications and the APACHE II score.

Neurologic (56.7\%) and cardiovascular (43.3\%) syndromes were the only complications of hypertensive emergencies represented. Similarly, Vilela et al reported $58 \%$ of cerebrovascular lesions (mainly ischemic stroke) and $38 \%$ of cardiovascular lesions (APO) as the most prominent HTN-E in their study done in Brazil on hypertensive crisis profile [13]. Stroke was the most prevalent HTN-E syndrome recorded among geriatric patients in the YEC. More than half of the 30 patients studied $56.7 \%$ ( $n=17)$ had 
stroke, $23.3 \%$ ( $n=7)$ had acute pulmonary oedema, and $10 \%(n=3)$ had coronary ischaemia and myocardial infarction each. Few patients presented with both APO and Ml, and APO and coronary ischaemia. Similarly, a Brazilian study on clinical study of hypertensive crisis in the medicine ward by Manjhvar et al projected stroke as the second most prevalent (39\%) hypertensive emergency in the medicine ward studied [14]. Also, a Greek study by Varounis et al on cardiovascular hypertensive crisis reported the most prevalent HTN-E been acute pulmonary oedema $(30.9 \%)$ in line with our study, followed by stroke (22\%) and then myocardial infarction (17.9\%) [15]. This study was done on all patients brought to the emergency department with hypertensive emergencies. Contrary to Vilela et al, we found no case of hypertensive encephalopathy, which is currently becoming increasingly rare, due to better BP control and available therapeutic options [13].

Regarding symptoms, neurologic symptoms included an equal prevalence in migraines and hemiparesis $23.3 \%(n=7)$ each, unconsciousness $13.3 \%(n=4)$, blurred vision, aphasia, alteration of general state, and convulsions. These symptoms occurred in association, and were not exclusive. Heart-related symptoms included: severe thoracic pain $16.7 \%(n=5)$, dyspnoea $13.3 \%(n=4)$, oedema of the lower limbs $10 \%(n=3)$, and palpitations. These symptoms were not isolated, but occurred in association with each other. Asthenia was recorded in most patients, while vomiting and nose bleeds was recorded in few patients. They were all associated with neurologic and heart-related symptoms.
The APACHE II score has been widely accepted as a measure of illness severity. It has been shown to accurately stratify the risk of death in a wide range of disease states, and in different clinical settings (like in ICU patients).

The patient's distribution in the APACHE ॥ score intervals showed a higher concentration of $43.3 \%(n=13)$ in the first group (score of 3-10), which is different from the Pakistani study on APACHE ॥ score correlation with mortality and length of stay in an intensive care unit by Naved et al [12] where there were more patients in the second group (score of 11 20). The next most represented group was the second group (score of 11-20) with $36.7 \%$ ( $n=11$ ), and lastly the third group (score of $21-30$ ) with $20 \%(n=06)$. The fourth group (score of 31-40) and fifth group (score > 40) was not represented. APACHE II score > 40 indicates a very high probability of death in the initial 2472hours of ICU admission. Results of our study shows a mortality rate of $30 \%$ which is in line with the $8.9 \%-38.3 \%$ reported from various parts of the world (Knaus et al, 1985).

Results of our study also showed a significant association between APACHE II score and the risk of mortality. In each successive APACHE II score interval, the mortality rates were higher than the preceding one $17.7 \%$ in the first group, $27.3 \%$ in the second group and $83.3 \%$ in the third group). These findings confirmed the capability of this scoring system to stratify patients according to the degree of severity of their disease. Our results showed good correlation between the observed and predicted mortality for all the groups. In group I, patients with 
APACHE II score of 3-10; the observed mortality was $7.7 \%$, while the predicted mortality was $11 \%$. In group 11 , patients with APACHE II score of 11-20; the observed mortality was $27.3 \%$, while the predicted mortality was $35.5 \%$. In the next group, patients with APACHE II score of 21-30; the observed mortality was $83.3 \%$, while the predicted mortality was $70.3 \%$. Therefore, a higher APACHE \| score increases patient's mortality risk.

Hypertensive emergencies are more frequent in the elderly; this has been predicted by numerous studies done worldwide. Though age is a prominent risk factor for hypertensive emergency, it was not discussed here because our study did not include all the age groups. Increasing age and previous health status is known to be associated with mortality and poor outcome [12,13]. However, age ranged from 60-82years. This prospective, crosssectional study identified several potential risk factors of hypertensive emergencies namely the female sex, non-adherence to antihypertensive drugs, duration of hypertension, number of prescribed antihypertensive medications, presence comorbidities, and an unknown hypertensive state. However, there was a possibility of interaction between these potential risk factors giving to our small sample size. In this study, the proportion of women experiencing HTN-E was slightly higher 53.3\% ( $n=16)$ than men $46.7 \%(n=14)$. This could probably reflect a greater number of females in the hypertensive population as asserted by Pollack \& Rees [16], in USA on the study acute care and evaluation of hypertensive emergency; and Shao et al [8] in Tanzania on the profile of patients with hypertensive crises presenting to an urban emergency department of a tertiary hospital. Several previous studies found indications of an increased incidence of HTN-E in women. For instance, a study by Saguner et al [17], in Switzerland on risk factors promoting hypertensive crisis explained that more women suffered from somatoform disorders as compared to men, and that this disease is associated with non-adherence. Furthermore, Vilela et al, assert that morbidity and mortality rates associated with any level of BP are higher in women than in men as from menopause [13]. This may be justified by differences in haemodynamic pattern between men and women, because before menopause, women have a lower peripheral vascular resistance and lower BP levels than men of the same age. After menopause, however, the female haemodynamic profile is not significantly different from the male profile in regard to BP [13], Therefore, women show an increased susceptibility to organic lesions after menopause, especially to cerebrocardiovascular diseases, as found in our case series. On the other hand, other studies have revealed a higher prevalence of HTN-E in men, namely: Vilela et al [13], and Tisdale et al [18],, a British study on the risk factors for hypertensive crisis. The greater number of HTN-E among men suggests that they are more susceptible to target-organ lesions than women. For instance, studies have shown that, the incidence of coronary artery disease in men increased in an almost linear mode as age increased.

Previous retrospective studies have shown that non-adherence to antihypertensive medications predisposes patients to hypertensive emergencies. Out of the 30 patients studied, $60 \%(n=18)$ were non- 
adherent to their antihypertensive medications, while $26.7 \%$ ( $n=8$ ) never consumed any antihypertensive medications. Similarly, a longitudinal study conducted by Saguner et al [17], revealed that $76.9 \%$ of patients with hypertensive crises were non-adherent to their antihypertensive therapy. Also, Tisdale et al [18], and an American study on diagnosis and treatment of hypertensive crisis in the elderly patients by Joseph \& Neil [19], supported that non-adherence to antihypertensives is an independent risk factor for hypertensive emergencies. Nevertheless, nonadherence was significantly associated with a higher number of prescribed antihypertensive medications as revealed by Pollack \& Rees, [16], Maria and al [20] on an update on hypertensive emergencies and urgencies in Italy. Therefore, this study suggests that a higher number of prescribed antihypertensive medications are not a true risk factor for HTN-E, but related to HTN-E through non-adherence.

Comorbidities were risk factors for hypertensive emergencies in approximately $70 \%$ of patients. Diabetes mellitus accounted for approximately $23.3 \%$ ( $n=7$ ), respiratory diseases $16.7 \%$ ( $n$ = 5) and other comorbidities like sepsis, ascites, obesity, HIV, alcoholism and tobacco abuse $30 \%$ ( $n=9)$ of all comorbidities. This was similar to the $26 \%$ recorded from patients with hypertensive emergencies by Vilela et al [13]. The prevalence of HTN-E in diabetics (40-50\%) is quite higher than in non-diabetics (20\%). Although hypertensive disease is multifactorial, the aetiological link between insulin resistance and hypertension becomes greater.
Metabolic abnormalities

(hyperglycaemia, hyperinsulinaemia, and dyslipidaemias) may play a role in the pathogenesis and complications of hypertension [13]. The vascular endothelium of diabetics shows a reduced synthesis of vasodilators and an increased release of procoagulants and vasoconstrictors. These defects coexist in hypertensive individuals, and could explain the greater incidence of atherosclerosis and hypertension. Diabetes mellitus is one of the major risk factors associated with the development of cerebrovascular (ischaemic stroke) and cardiovascular (ischaemic coronary disease) complications [16]. These data may be correlated with our case series of $56.7 \%$ of cerebrovascular lesions and $43.3 \%$ of cardiovascular lesions. Hypertensive emergencies occur in some patients who do not report a history of hypertension, or previous use of antihypertensive medications. Our study revealed up to $20 \%(n=6)$ of patients who neither reported a history of hypertension, nor used any antihypertensive medications. Likewise, Varounis et al [15] showed that hypertensive emergencies occurred in $23 \%$ of patients without prior history of hypertension or antihypertensive use.

Out of the 30 patients studied, $70 \%$ ( $n=$ 21) were discharged. In addition, $50 \%$ ( $n=$ 15) of patients had no complications, while the remaining $50 \%(n=15)$ had at least one complication (neurologic, cardiac or vascular disabilities). Prominent among these complications were neurologic complications which accounted for 23.3\%, followed by cardiac complications $16.7 \%$ and the least represented were vascular 
complications, $10 \%$. The neurologic complications were: gait disturbance, hemianopia and seizures. These were in line with the neurologic disabilities recorded by Jauch et al in a USA [21] stroke unit, in a study titled: early management of acute ischaemic stroke. The incidence of seizures after ischaemic infarction is < 10\% [22]. About cardiac cmplicatiopns :cardiac arrhythmias, and cardiogenic shock, in line with the findings of Jauch et al [21] in a USA specialised stroke unit. DVT and suspected PE (10\%) were the main vascular complications registered in our study, and they were predominantly due to stroke. Pulmonary emboli generally arise from venous thrombi that develop in a paralysed lower extremity or pelvis [21]. Hemiparesis accounted for $23.3 \%$ of stroke symptoms recorded in our study, hence the complications of PE and DVT. These complications resulted in significant disability and mortality. Similar findings were also described by Jauch et al [21], where PE accounted for $10 \%$ of deaths following stroke, and about $1 \%$ of complications due to stroke, and DVT accounted for $2.5 \%$ of stroke complications. A polish study by Indredavik et al [23], titled medical complications in a comprehensive stroke unit and an early supported discharge in Norway found PE in $<2.5 \%$ of patients during the first week in a specialized stroke unit. Besides been associated with a life threatening pulmonary event, DVT slows recovery and rehabilitation after stroke. The risk of DVT is highest among critically ill, immobilised and older patients with severe stroke. A study on venous thromboprophylaxis in critical care by the intensive care society in America reported the post mortem detection of $\mathrm{PE}$ as high as $27 \%$ and DVT during ICU hospitalisation ranging from < $10 \%$ to almost $100 \%$ depending on the screening methods and diagnostic criteria used.

Our findings revealed a very high mortality rate of $30 \%(n=9)$ out of the 30 patients studied. This is comparable with a recent study done in the same center by Hamadou et al [24], in a study titled clinical presentations and outcome of cardiovascular emergencies in Yaoundé, where geriatric hypertensive emergencies had a poor prognosis, and hypertensive emergencies as in all age groups accounted for $10-15 \%$ deaths. Our findings were different from the Greek study by Varounis et al [15] who recorded a $4.6 \%$ mortality rate due to hypertensive emergencies in all age groups.

Patients who died (30\%) had at least one comorbidity $(70 \%)$ associated with their hypertensive emergency, higher APACHE II scores and more disabilities (50\%). All the patients studied had at least one risk factor that triggered the HTN-E. This mortality rate was quite high, despite the promptness in treatment of these patients within one hour of hospitalisation and a wide availability of appropriate treatment strategies. Mortality was predominantly caused by cardiovascular deficits (77.8\%), higher APACHE II scores, very elderly patients (44.4\%) and comorbidities (77.8\%) and non-adherence to antihypertensives (66.7\%). Despite the lower prevalence of the middle-aged and the aged patients, the mortality rate recorded in them was quite high as half of the patients in these two groups died. This goes a long way to explain a higher mortality rate in more elderly patients, compared to the young old patients, 
probably due to physiological changes associated with aging among other health factors (hypertensive emergency syndromes, APACHE $\|$ score and risk factors). Therefore, age alone is inadequate to predict patient's evolution. Moreover, the little prevalence of very elderly patients in our study could be as a result of the low life expectancy in Cameroon.

\section{Conclusion}

The hypertensive emergency syndromes encountered were neurologic deficit (stroke $56.7 \%$ ) and cardiovascular deficits (43.3\%). They were associated with a substantial morbidity rate of $50 \%$. These syndromes were detected with the help of predominant signs and symptoms in order of prevalence such as: migraine, hemiparesis, sever thoracic pain, unconsciousness, dyspnoea, and pedal oedema among others; and then later on confirmed using laboratory test results. Higher APACHE II scores were quite effective in predicting mortality among these patients, and they were associated with higher mortality rates, while lower APACHE II scores were associated with lower mortality rates. For an APACHE ॥ score of 21-30 for instance, the observed mortality was $83.3 \%$ while the predicted mortality was $70.3 \%$. Hence, direct proportionality between the APACHE ॥ score and the prediction of mortality rates. The main triggering factor for hypertensive emergency was nonadherence to antihypertensive therapy or inappropriate medical treatment, as $86.3 \%$ of patients were noncompliant. Other risk factors among others comprised in: comorbidities $170 \%$ diabetes mellitus, respiratory diseases and others), unknown hypertensive status (20\%), and the female sex (53.3\%). All the health factors studied contributed to mortality as follows: neurologic deficit $22.2 \%$, cardiovascular deficits $77.8 \%$; APACHE II score: group I, 7.7\%, group II, $27.3 \%$, and group III, $83.3 \%$; the young-old patients $55.6 \%$, the middle-aged old and aged patients $44.4 \%$; comorbidities $77.8 \%$; non-adherence to antihypertensives $66.7 \%$; the female sex $55.6 \%$, the male sex $44.4 \%$; and an unknown hypertensive status $33.3 \%$. Therefore, a recovery rate of $70 \%$ and a high mortality rate of $30 \%$ was recorded, alongside a high incidence (50\%) of complications. This high mortality rate was predominantly due to cardiovascular deficits $77.8 \%$, APACHE || score group || $83.3 \%$, the young-old patients $55.6 \%$, comorbidities $77.8 \%$ non-adherence to antihypertensives $66.7 \%$, and the female sex $55.6 \%$. Hypertensive emergencies remain a public health problem in developing countries.

\section{Références}

1- Nikolaos, L., Dimitrios, M., Elias, S., Georgios, F., Maria, G. Hypertension in the elderly. World Journal of Cardiology 2012, 4 (5) : 135-147

2- Mohamed A. Al-Modee, Noha S. Hassanien, and Chauky M. Jabloun. Profile of morbidity among elderly at home health care service in Southern Saudi Arabia. J Family Community Med. 2013 Jan-Apr; 20(1): 53-57. 
3- Salma, M. S. Geriatrics intensive care unit: outcome and risk factors for in hospital mortality. Advances in aging research 2013, 2 (4): 166-169.

4- Ouchi, Y., Rakugi, H., Arai,H., Akishita, M., Ito, H., Toba, K. et all. Definition and classification of the Elderly by the Joint committee of Japan Gerontological Society (JGS) and Japan Geriatric Society (JGS). Geriatrics and Gerontological International 2017, 17(2). doi.org/10.1111/ggi.13118.

5- Internists' and intensivists' roles in intensive care admission decisions: a qualitative study. Stéphane Cullati, Patricia Hudelson, Bara Ricou, Mathieu Nendaz, Thomas V. Perneger et all. BMC Health Services Research 2018, $18: 620$.

6- Varon, J., Marik, P. Clinical review: the management of hypertensive crises. Critical Care Journal 2003, 7 (5): 374 384. doi: $10.1186 / /$ cc2351.

7- Tabi, A., Dimala, A., Atashili, J., Mbuagbaw, J., Monekosso, G. International Journal of Hypertension 2016. doi: 10.1155/2016/5639146

8- Shao, P., Sawe, H., Murray, B., Mfinanga, J., Mwafongo, V., Runyon, M. Profile of patients with hypertensive urgency and emergency presenting to an urban emergency department of a tertiary referral hospital in Tanzania. BMC Cardiovascular Disorders 2018, 18: 158. https://doi.org/10.1186/s12872-0180895-0.

9- Joseph, V., \& Neil. E. Diagnosis and treatment of hypertensive crises in the elderly patients. Journal of Geriatric Cardiology 2007, 4 (1): 103-107.
10-William J. E. Hypertension : a companion to braunwald's heart disease 2018, 3rd ed. P427-432.

11-Varounis, C., Katsi, V., Nihoyannopoulos, P., Lekakis, J., Tousoulis, D. Cardiovascular hypertensive crisis: recent evidence and review of the literature. Frontiers in Cardiovascular Medicine 2017, 3: 51. doi: 10.3389/fcrm.2016.00051.

12-Naved, S., Siddiqui, S., Khan, F. APACHE-II score correlation with mortality and length of stay in an intensive care unit. Journal of the College of Physicians and Surgeons Pakistan 2011, 21 (1): 4-8.

13-Vilela, M., Higashiama, W., Garcia, E., Luizon, M., Cipullo, J. Hypertensive crisis profile. Prevalence and clinical presentation. Arquivos Brasileiros de Cardiologia 2004, 83 (2): 131-136.

14-Manjhvar, S., Thakare, S., Gupta, H., Indurka, M. Clinical study of hypertensive crisis in medicine ward. International Journal of Contemporary Medical Research 2017, 4 (11): 24547379.

15-Varounis, C., Katsi, V., Nihoyannopoulos, P., Lekakis, J., Tousoulis, D. Cardiovascular hypertensive crisis: recent evidence and review of the literature. Frontiers in Cardiovascular Medicine 2017, 3: 51. doi: 10.3389/fcvm.2016.00051.

16-Pollack, C., Rees, C. Hypertensive emergencies: Acute care evaluation and management. Emergency Medicine Cardiac Research and Education Group, EMCREG 2008,5 (3): $1-8$

17-Saguner, A., Dur, S., Perrig, M., Schiemann, U., Stuck, A., Burgi, U., Erne, P., Schoenenberger, A. Risk factors promoting hypertensive crisis: 
evidence from a longitudinal study.American Journal of Hypertension 2010, 23 (7) : 775-780

18-Tisdale, J., Huang, M., Borzak, S. Risk factors for hypertensive crises: importance of out-patient blood pressure sontrol. Family Practice 2004, 21 (4): 420-424

19-Joseph, V., \& Neil. E. Diagnosis and treatment of hypertensive crises in the elderly patients. Journal of Geriatric Cardiology 2007,4 (1):33-47

20-Maria, L., Massimo, S., Valentina, A., Salvatore, S., Stefano, P., Andrea, S. et al. An update on hypertensive emergencies and urgencies. Journal of Cardiovascular Medicine 2014, 16 (5) :102

21-Jauch, E.,Saver, L., Adams, H.,Askiel, B., Connors, J., Wang, Z. et al. Guidelines for the early management of acute ischaemic stroke 2013. Stroke, 44:870-947
22-Alberti, A., Paciaroni, M., Caso, V., Venti, M., Palmerini, F., Agnelli, G. Early seizures in patients with acute stroke: frequency, predictive factors, and effects on clinical outcome. Vascular Health Management 2008. 4: 715-720

23-Indredavik, B., Rohweder, G., Naalsund, E., Lydersen, S. Medical complications in a comprehensive stroke unit and an early supported discharge service. Stroke 2008, 39: 414420

24-Hamadou, B., Kalissou, Y., Jingi, A., Amougou, S., Dikosso, S., Njouoguep, R., \&Kingue, S. Clinical presentations and outcome of cardiovascular emergencies in Yaoundé: a crosssectional study. World Journal of Cardiovascular Diseases 2018, 8 (2): 133-142 